\title{
Digital twin-based opti-state control method for a synchronized production Check toroperation system
}

Kai Zhang, Ting Qu, Dajian Zhou, Hongfei J iang, Yuanxin Lin, Peize Li, Hongfei Guo, Yang Liu, Congdong Li and George Q. Huang

The self-archived postprint version of this journal article is available at Linköping University Institutional Repository (DiVA):

http:// urn.kb.se/ resolve?urn=urn:nbn:se:liu:diva-164189

N.B.: When citing this work, cite the original publication.

Zhang, K., Qu, T., Zhou, D., Jiang, H., Lin, Y., Li, P., Guo, H., Liu, Y., Li, C., Huang, G. Q., (2020), Digital twin-based opti-state control method for a synchronized production Check toroperation system, Robotics and Computer-Integrated Manufacturing, 63, 101892.

https:// doi.org/ 10.1016/j.rcim.2019.101892

Original publication available at:

https:// doi.org/ 10.1016/j.rcim.2019.101892

Copyright: Elsevier

http:// www.elsevier.com/

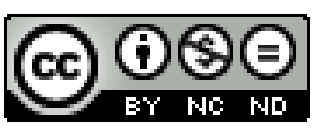




\title{
Digital twin-based opti-state control method for a synchronized production operation system
}

(i) The corrections made in this section will be reviewed and approved by journal production editor.

Kai Zhang ${ }^{\mathrm{a}, \mathrm{c}}$, Ting Qu ${ }^{\mathrm{b}, \mathrm{c}, *}$ quting@jnu.edu.cn, Dajian Zhou ${ }^{\mathrm{d}}$, Hongfei Jiang ${ }^{\mathrm{a}, \mathrm{c}}$, Yuanxin Lin ${ }^{\mathrm{a}, \mathrm{c}}$, Peize Li ${ }^{\mathrm{c}, \mathrm{e}}$, Hongfei Guo ${ }^{c}$, Yang Liu ${ }^{c, f}$, Congdong Li ${ }^{\mathrm{a}, \mathrm{c}}$, George Q Huang ${ }^{\mathrm{c}, \mathrm{g}}$

${ }^{a}$ School of Management, Jinan University, Guangzhou 510632, China

${ }^{\mathbf{b}}$ School of Intelligent Systems Science and Engineering, Jinan University (Zhuhai Campus), Zhuhai 519070, China

${ }^{\mathbf{c}}$ Institute of Physical Internet, Jinan University (Zhuhai Campus), Zhuhai 519070, China

dGuangdong CIMS Provincial Key Lab, Guangdong University of Technology, Guangzhou 510006, China

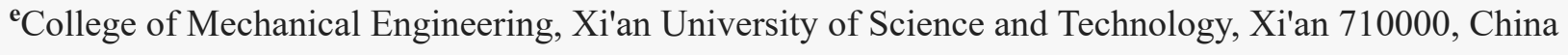

f Department of Management and Engineering, Linköping University, SE-581 83, Linköping, Sweden

gepartment of Industrial and Manufacturing Systems Engineering, The University of Hong Kong, Hong

Kong, China

*Corresponding author at: School of Intelligent Systems Science and Engineering, Jinan University (Zhuhai Campus), Zhuhai 519070, China.

\begin{abstract}
The intelligent manufacturing strategy and customer demand have mutually promoted each other. Also, the production mode is shifting towards customized production, and more rental resources or services are introduced to the production system, therefore, the systems are becoming more complex. Compared with traditional production systems, such systems have some new features, this work calls this type of system as a synchronized production operation system (SPOS). Under such circumstances, production systems are influenced by more frequent uncertainties, and the planningbased production decision and control approach is no longer applicable. The opti-state control (OsC) method is proposed to help SPOS keep in an optimal state when uncertainties affect the system. Besides, a digital twin-based control framework (DTCF) is designed for getting the full
\end{abstract}


element information needed for decision making. Based on the comprehensive information of the production system obtained by the $\mathrm{DTCF}$, the $\mathrm{OsC}$ method is introduced to the virtual control layer to formulate the optimal target guiding the path of the system in real time through the dynamic matching mechanism (qualitative perspective). Then multi-stage synchronized control with analysis target cascading (ATC) method is used to get the local optimal state decisions (quantitative perspective). From both qualitative and quantitative aspects to ensure the system is under an optimal target path for optimal operation procedure. At last, a case study in a large-size paint making company in China is used to validate the effectiveness of the approach.

Keywords: Optimal State; Digital Twin; Resilient Control; Synchronized Control; Uncertainties

\section{Introduction}

With the development of the social economy, the customer requirement is shifting towards the pursuit of individualized needs, and the production mode is also gradually shifting from mass production to multi-variety \& small-batch mode [1]. Driven by intelligent manufacturing strategies or solutions, such as Industrial Internet, Industry 4.0, and Made in China 2025, various advanced software and hardware enabling technologies and management methods are widely used in various production systems, such as cyber-physical system (CPS), digital twin (DT) and industrial product service system [2]. In order to meet the high-frequency variation of the production tasks formed by customized and personalized requirements, the software and hardware resources should operate and cooperate autonomously, flexibly, agilely and rapidly. In this work, a synchronized production operation system (SPOS) is defined to describe a customized production operation system supported by an open resource system. Due to the randomness of customer orders, the dynamic of production demand, the service of external resources and the low repeatability of production process, the SPOS can be disturbed inevitably by dynamic interference from orders, resources, materials, quality, service and so on. It is hard to complete the actual tasks according to the preset capabilities and optimization goals for highly automated manufacturing equipment or precisely optimized manufacturing processes. This situation is exacerbated by the level of product customization and the complexity of manufacturing systems.

Therefore, how to maintain the performance of production system in the optimal state under dynamic interference has always been a hot topic for scholars in this field. In order to keep the SPOS in an optimal state when uncertainties acts on the system, there are two major research questions need to be solved: 1) How to design a reasonable information framework to obtain the full element information and operation status data of the execution layer and establish the real-time operation model. 2) How to make the decision-making layer of the system construct the decision model quickly and adaptively under dynamic interference, then make the decision beneficial to the optimal operation of the system.

The biggest challenge for the first question is how to get the SPOS operational status data as completely and accurately as possible, then process the data based on a series of constraints, models and algorithms to reflect the real-time operational status of the SPOS, thus providing comprehensive information support for decision making. Digital twin (DT) is a virtual model that creates physical entities in a digital world, using data to 
simulate the behavior of physical entities in real-world environments, and adds or expands new capabilities for physical entities through virtual interactive feedback, data fusion analysis, decision iteration optimization, and other means [3].

In addition, for the second question the challenge is how to construct a real-time information based adaptive and systematical decision mechanism and algorithm to keep the SPOS operate in optimal state. Resilient control is a system control theory aims at restoring the system performance to the original state through dynamic elimination and operation adjustment when production process is disturbed by uncertainty factors [4]. However, resilient control for SPOS cannot solve the impact of random uncertainty factors on the system, and it is difficult to judge whether the original state is suitable for the current operating state of the system, also it is difficult to ensure that the original state is the optimal choice for the system.

In order to maintain the performance of production system in the optimal state under dynamic interference an Opti-state control (OsC) method is proposed in this work. Based on the idea of resilient control, OsC aims to keep the system in optimal operation state, not only to make decisions based on the goal of reducing the impact of a certain dynamic on the system. In order to make this method practical, a digital twin control framework (DTCF) is also proposed to help decision-making layer get full element information of execution status from all units in the execution layer. DTCF also has an interface to import various decision methods including $\mathrm{OsC}$, to simulate or predict all kinds of decisions in real time, and finally the best decision is given to the physical layer to guide the system.

The remainder of this paper is organized as follows: Section 2 reviews some previous studies relevant to the proposed DTCF and OsC. Section 3 analyzes the problems of this study and briefly introduces the basic of SPOS. Section 4 proposes the two technical solutions which are DTCF and OsC. In Section 5, a case study to test the effectiveness of OsC is presented. Final conclusions are summarized in Section 6.

\section{Literature review}

In order to solve the research questions addressed in this paper, two aspects need to be studied, which is realtime information management and decision methods.

\subsection{Real-time data management method \& Information system framework}

\subsubsection{Real-time management of production systems}

In recent years, scholars have conducted extensive research and practice on the application of the Internet of Things (IoT) technology in the manufacturing industry, gradually expanding from the initial production or logistics units to the multiple links of the entire production system. IoT provides a reliable solution for realtime information acquisition and monitoring of dynamic production processes [5]. Qu et al. used dynamic information captured from IoT systems to analyze the operations of internal/external production systems [6]. Ren et al. conducted a comprehensive overview of big data in smart manufacturing and proposed a conceptual framework from the perspective of product lifecycle [7]. To deals with the social manufacturing trend, Ding proposed an RFID-enabled social manufacturing system (RFID-SMS) to realize the real-time monitoring and dispatching of inter-enterprise production and transportation tasks [8]. Zhong et al. applied real-time 
manufacturing execution systems for mass-customized production, real-time and accurate data from the shop floor can be uploaded to a level and displayed to decision makers to develop different strategies [9]. In addition, Parikhab et al. used these real-time data to guide the planning and design of storage shelves [10]. Zhang et al. designed a cyber-physical system-based smart control model for shopfloor material handling [11]. Based on real-time manufacturing data, an improved autonomous manufacturing task orchestration model is proposed to make it more practical to predict dynamic machine sequence [12].

\subsubsection{Digital Twin}

The concept of digital twins was first proposed by professor Grieves from the university of Michigan in his product lifecycle management course. Since the concept of digital twin was put forward, the academia has carried out related research in the main aspects of digital twin modeling $[13,14]$, interaction and synergy $[15$, 16], service application $[17,18]$. The workshop is the basis for the execution of manufacturing activities, and scholars have conducted a lot of research around the digital workshop.

Tao and Zhang et al. proposed the concept of digital twin workshop and expounded its system composition, operation mechanism, characteristics, and key technologies [19]. Zhang et al. et al. proposed a framework for a future intelligent workshop, which provides a feasible method for designing an autonomous workshop with abnormal processing capabilities [20]. Ding built the interconnection and interoperability of a physical shop floor and corresponding cybershop floor using cyber-physical system (CPS) and digital twin technologies, then established a digital twin-based Cyber-Physical Production System (DT-CPPS) expected that DT-CPPS can provide the basis for shop floors [21]. Zhuang et al. discussed the future work to implement digital twin-based smart production management and control concept for complex product assembly shop-floors [22]. Söderberg et al. applied digital twin to production process control, optimizing the tolerances, positioner positions, clamping strategies, welding sequences, etc. of the product during design and pre-production [23]. Coronado et al. built digital twin workshops for data production control and optimization [24]. Tao et al. summarized the progress of digital twin in enterprise application and theoretical research, and proposed the five-dimensional structural model of digital twin and six application criteria for digital twin driving, and explored the key issues and technologies that need to be addressed in the 14 categories application scenarios and implementation driven by digital twin, which provided a theoretical and methodological reference for further application of digital twin in the future $[3,25,26]$.

\subsection{Decision and control approach for operational process}

\subsubsection{Decision and control method}

Resilient control is an emerging control method, which means that the system can tolerate fluctuations caused by its structural design parameters, control device structure, and parameters. The purposes of resilient control are to minimize the probability of occurrence of adverse events or allow events that have occurred to be mitigated or restored to normal levels in a short time [27]. Resilient control has been applied in the fields of social management, infrastructure, and industrial systems [28, 29, 30]. Jin et al. proposed a comprehensive robustness and elastic control strategy to improve the operational safety and performance of nuclear power plants [31]. In order to closely monitor and control complex, high-performance engineering systems, Lin et al. 
developed the resilient monitoring and control (ReMAC) system [32]. Wang et al. discussed the concept of resilient scheduling for manufacturing systems and designed a three-layer resilient scheduling paradigm to protect the manufacturing system from dynamics [33]. Woods groups four different basic concepts using of the label resilience: (1) rebound resilience; (2) robustness resilience; (3) graceful extensibility resilience; (4) sustain adaptability resilience and sum up how scholars apply resilience control in the four concepts mentioned above [34]. However, there are few studies on the management and control of industrial systems, especially online management and control.

Multidisciplinary design optimization (MDO) is a design for large-scale complex engineering systems composed of many interacting subsystems that have been widely used in many fields with complex engineering systems. Typical multidisciplinary design optimization methods include concurrent subspace optimization (CSSO), Bi-level integrated system synthesis (BLISS) methods, collaborative optimization (CO) and analysis target cascading (ATC). Although the CSSO and BLISS methods can effectively solve the severe coupling between subsystems, the convergence is not strictly theoretically proven, and the calculation is large. The CO method has a high calculation speed but is strictly limited to two-level optimization. The decomposed analysis model must be on the same level. Otherwise, the convergence is difficult to guarantee, and the complex system is usually multi-level. At the same time, complex systems are often multi-layered. ATC has the advantages of parallel optimization, unrestricted number of levels, and strict convergence proof for solving complex large system optimizations that can be decomposed into hierarchical architectures.

\subsubsection{Synchronization decision and mechanism for production system}

In recent years, it has been quite common for scholars to use integrated optimization methods to solve production coordination decision-making problems among production workshops, distribution fleets, and warehouses. Most of them consider the multiple-links static collaborative decision-making problem of production systems in complex environments. Monostori et al. based on distributed operational and control theory, establishes an enterprise-level production and logistics collaboration model through the integration of cooperative game theory and distributed machine learning theory [35]. Bard et al. studied the integration of "production-distribution-path" in multi-processing centers, multi-products, and single distribution centers [36]. The methodological framework proposed by Liotta et al. supported the optimization of the production logistics with detailed dynamic distribution plans affected by demand uncertainty [37]. A production logistics system is often subject to high operational dynamics due to large working areas, frequent resource interactions and long operation periods [6]. To Reduce the impact of the dynamics that occur in production-logistics systems, Lei and $\mathrm{Qu}$ et al. proposed an IoT-based real-time production logistics synchronization(PLS) system under cloud manufacturing environment, which enables a smart PLS mechanism with two-level dynamics control [38]. Qu et al. proposes the TPS synchronization control mechanism by study the synchronization decision-making and control problem between the "production-transport-warehousing" multiple-links in the production-logistics system under the interference of dynamic factors [39]. Luo et al. proposes an Ubi-SPL (Synchronized Production and Logistics via Ubiquitous Technology) framework using the ubiquitous computing as the enabling technology by analyzing the requirements and challenges in real life industry [40]. In addition, Zhang proposed an extended ALC approach to cloud manufacturing to address manufacturing service configuration issues, which considering the decision autonomy and limited production capacity of service providers [41]. A 
data-driven model based on analytical target cascading is proposed to achieve the self-organizing configuration of collaborative production-logistics by Zhang [42].

\subsection{Summary of literature}

From the literature review, it can be found that lots of research on real-time information management mostly stays in local data visualization applications. On the other hand, applications in production systems are mostly limited to coarse-grained static integration optimization. DT framework could acquire data in physical word comprehensively, accurately and quickly. In addition, it mainly stays at the level of precise mapping and simulation for specific objects and specific work content. However, there are few studies on the application of digital twin to the systematic modeling of large systems with complex dynamic structures and the iterative management for system lifecycle.

From decision \& control methods aspect, the method proposed by most scholars is based on problem-solving, that is, passive response dynamics to reduce its impact on system operation. On the other hand, limited research on the decision-making control methods aiming at maintaining the optimal operation of the system.

Therefore, this work proposes an optimal state control concept and decision-making method, which combining the real-time environment and state to adaptively determine an optimal operational target, then optimize the decision based on the new target to keep the system stay in an optimal operation state.

\section{Problem Formulation}

\subsection{The operation mode of SPOS and its characteristics}

We abstract the modern complex production system that based on open production resources and lean management with high degree of informatization as synchronized production operation system.

\subsubsection{Typical SPOS operation mode}

The SPOS is based on a typical production operation system. (shown in Fig. 1). The SPOS is surrounded by IoT devices, and the system includes multiple operating units that can make independent decisions, such as customer service center, production unit, plant logistics unit, warehousing unit and transportation unit. Each unit can be divided into multiple agents (shops, fleets or warehouses). SPOS based on open resource system, every unit not only holds its own resources, but also uses external resources, such as cloud resources or other rental resources. Service outsourcing, such as 3PL is also part of the open resource system.

alt-text: Fig 1

Fig. 1 


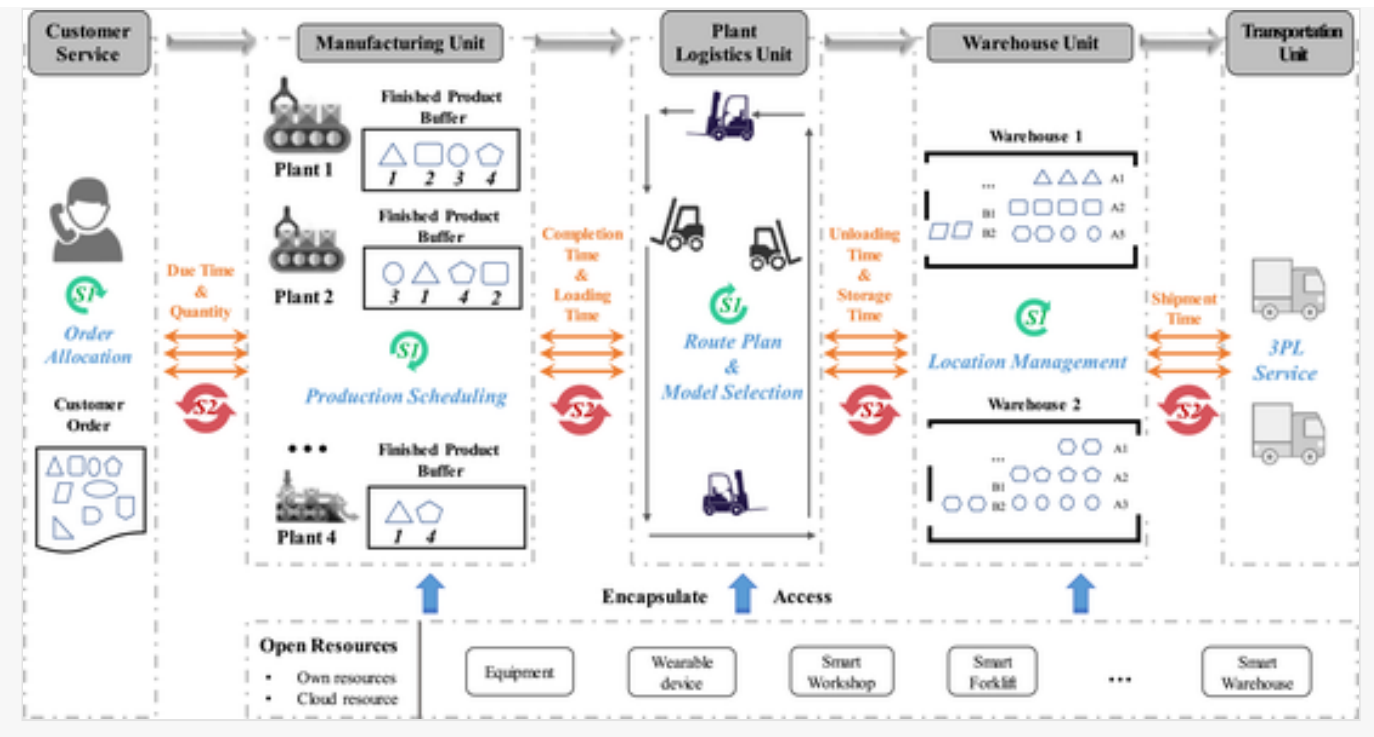

Typical SPOS operation process.

The typical operation process of SPOS is as below: The customer service center receives customized orders and assigns the enterprise-level production tasks to the production execution units. Every unit of the system makes plans based on the current (before starting production) of the overall resource occupancy status, resource rental requirements, order demand and the plan of correlative units, which include resource plan, production plans, transportation plans, warehousing plans, etc. When the plan is executed, production unit produces the products according to the production plan, and temporarily storing the finished products at the finished production buffer waiting for the plant logistics fleet (e.g. forklift truck) to be transported. The transport fleets unit transport the finished product to the finished product warehouse according to the product attributes, completed time, and transportation schedule. The warehouse unit assigns the finished product storage according to the pre-plan for location allocation. Then complete the order shipment task before the due date. All kinds of decisions are based on the operational status of the relevant units to make synchronized decisions, thus ensuring that the SPOS is in a state of synchronization operation throughout the operation process,

The SPOS synchronized operation is the synchronized behavior of the production system for the three main stages of the product life cycle. There are three main aspects: S0) In the system design phase, dynamically introduce external resources and design system configuration for customized product functional requirements, and adopt adaptive design to obtain adaptive production structure; S1) In the production planning phase, independent production units (subsystems) with heterogeneous decision structures and control objectives in customized production systems are used for linkage decision making to develop coordinated production plans; S2) In the production execution phase, according to the diversity interference presented by the customized production process, the online synchronized control between the production subsystems/units in the coupling operation process is carried out to ensure the realization of key production operation goals. This paper mainly analyzes the synchronization between the planning phase and the execution phase.

\subsubsection{The general production systems and SPOS}


The general production system is an extensive management mode, the top-level design of the upper planners is used to coordinate and control the operation of each decision-making unit. The association relationship of each decision-making unit is a kind of canonical relationship with low degree of association, which may result in low system control precision and high cost.

In highly variable production systems, the dynamics of correlation between decision-making units are enhanced due to increased uncertainty in various manufacturing resources. This leads to a relationship between decision-making units that is not as standardized and long-term as a general production system. General production system is hard to meet the needs of multi-variety small-batch manufacturing.

Synchronized production operation system is a JIT-type synchronized production system that can establish the dynamic association of each unit in real time under a highly dynamic production environment. SPOS includes multiple independent decision-making, control, and operational decision-making units with operational or structural dynamic relationships, which can efficiently and effectively respond to dynamic disturbances at various levels. SPOS is a refined management mode, which effectively meets the needs of multi-variety and small- batch.

The specific differences between the general production system and SPOS are shown in Table 1.

\section{alt-text: Table 1}

\section{Table 1}

The general production systems and SPOS.

\begin{tabular}{|l|l|l|}
\hline Comparison item & General production system & SPOS \\
\hline Decision-making unit number & Multiple units & Multiple units \\
\hline Units association relation & Normative and long-term & Dynamic association \\
\hline Responsivity & Low responsivity & High responsivity \\
\hline Control precision & Low control precision & High control precision \\
\hline Decision method & All in one decision & Distributed decision \\
\hline Optimize target & Local control and optimization & Global control and optimization \\
\hline
\end{tabular}

\subsection{Uncertainties analysis for SPOS}

Uncertainties refers to the lack of accurate knowledge or prediction of a process or parameter [43]. Uncertainty mainly comes from lack of information, complexity of information, evidence of conflicting conflicts, 
ambiguity of description, measurement error and reliability of information [44]. At present, there are many works of literatures that classify uncertainties. Subrahmanyam et al. divides uncertainties into short-term uncertainties and long-term uncertainties [45]. Based on the nature of the source of uncertainty in a process, Pistikopoulos divides uncertainties into four categories: model uncertainties, process uncertainties, external uncertain factors, and discrete uncertain factors based on the nature of the source of uncertainty in a process [46]. Bräutigam et al. divided uncertainties into endogenous uncertainties and exogenous uncertainties. Endogenous uncertainties, such as equipment operation and task processing speed, etc., are constantly changing uncertainties related to specific internal enterprises, including uncertainty factors related to time, complexity, available cost, current capital, product quality and characteristics. Exogenous uncertainties refer to uncertainties from outside the company (marketing), including uncertainties in market competition, market volatility, and policy impact [47].

The general optimization goal of a production system consists of three types of elements: time, quantity, and cost. In order to meet the production target, it is necessary to satisfy all the elements of the production system (such as equipment, employees, etc.) according to the established parameters. It leads to two types of uncertainty:

- Element uncertainty factors

Uncertainties in the elements of the production system, such as machine failures, physical state of the employee. Under such dynamics, the system can keep its original target generally. At this time, with a certain control method, it can make the system less affected by the dynamics, and help the system restore the system to the original state, and this process is the idea of resilient control.

- Target uncertainty factors

Uncertainties on the target of the production system, such as changes in order quantities, changes in delivery dates, etc. Since such uncertainty changes the target of the system, it is difficult to directly use the method of resilient control method. At this point, a new control method is needed, which combines the uncertainties to redetermine the system target and gives an updated execution plan to guide the system to the updated target.

In the actual production system operation process, every independent unit or correlated units of the SPOS may encounter different uncertainty factors. Because of the characteristics of uncertainties, it is difficult for the SPOS to predict the timing of uncertainty, which makes it more difficult for the decision-making layer to make a real-time and accurate decisions. Hence, it is difficult for the system to operate in an optimal state.

\subsection{Challenge analysis}

Through the analysis of SPOS characteristics and the uncertainty factors, there are two challenges to achieving optimal control of SPOS:

- System uncertainties perception aspect: SPOS is based on open resources and its resource composition is complex. The execution layer contains a wide variety of resources, including a large amount of dynamic data such as employees, equipment, materials, vehicles and logistics. 
The acquisition of real-time dynamic information is an important support for realizing SPOS optimal control. Due to the lack of effective sensing approach, it is hard for the traditional production system to realizes the comprehensive perception and real-time monitoring of the dynamic information of the actual execution process, therefore the decision-making layer cannot get the real-time operating information of the system cannot to response the dynamic interference in time.

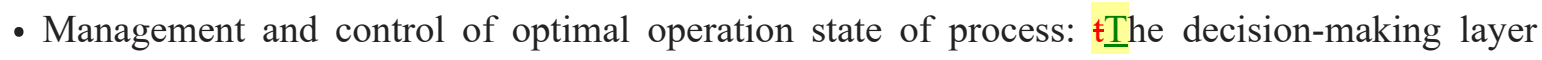
cannot obtain the execution status from the execution layer timely and accurately, so that the decision-maker cannot adjust the plan after the execution process is disturbed by uncertainty factors. On the other hand, the overall event-driven or static experience-based manage \& control mechanism lacks multi-dimensions and adaptive assessment of uncertainty factors in a specific state of the production system. Moreover, traditional decision method adopts centralized decision-making and decentralized execution way, which cannot adapt to the situation that the resources in the production system are changeable and the resource relationship is also changeable.

\section{Digital twin-based opti-state control framework and mechanism for SPOS}

Based on the problem analysis in the previous section, this section introduces the digital twin-based SPOS opti-state control method in details four parts. Firstly, introduce the digital twin-based control framework (DTCF) for SPOS. Then, the core technical, the opti-state control (OsC) concept, is proposed. Finally, it is introduced how to achieve optimal control of SOPS from qualitative and quantitative perspectives.

\subsection{Digital twin-based opti-state control framework for SPOS}

The purpose of OsC is to keep the system in optimal state. When uncertainties changed the environment, relationship and constraints, the decision-making layer makes an opti-state decision through a large-scale recalculation based on the data and model of the production system as complete as possible. In addition, digital twin can build a real-time complete model of the physical layer in the virtual layer, which provides a suitable information framework for optimal control. In order to better implement the OsC method, this paper proposes a digital twin control framework (DTCF) based on the general architecture of DT.

The life cycle of SPOS in a complex dynamic environment is divided into three phases: dynamic configuration, precise mapping and OsC. So, the virtual model layer which targeting the exact mapping of a physical object in the original DT concept is expanded to the virtual control layer. The virtual control layer acts on the control of the full lifecycle of the synchronization production system. DTCF needs imports the opti-state mechanism to form digital twin-based opti-state control framework (Fig. 2).

- Physical Object Layer 


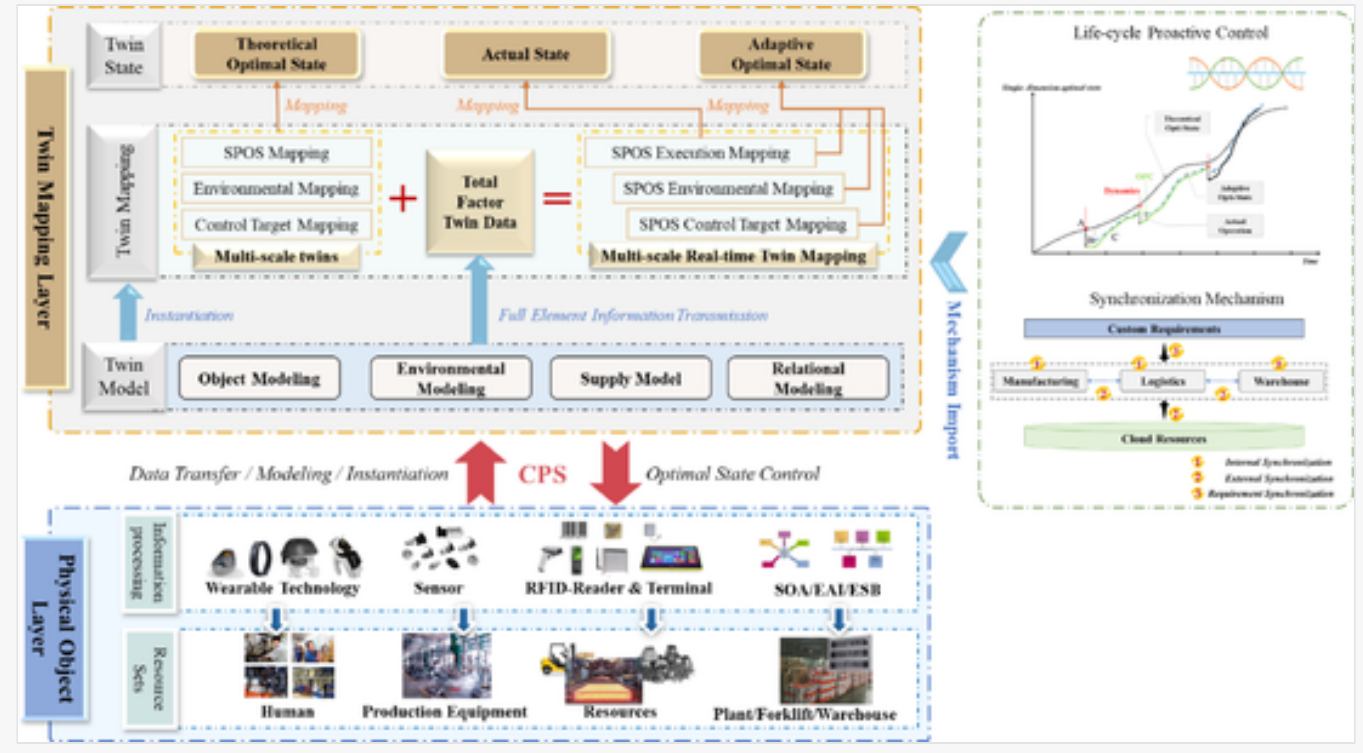

DT-based opti-state control framework.

The physical object layer is an objective entity set of the production system. It receives decision-making task instructions issued by various enterprise information systems. The information generated during the operation of SPOS can be collecting, sensing, processing and transmitted to provide basic data support for virtual control layer.

There are two sub-layers in physical object layer: (1) Resource sets layer: various resource entities of the production system, including entities such as workers, machines, objects, and units; (2) Information processing layer: making the production resources intelligence production resources and integrate and pre-process information.

The physical object layer includes not only the real time information of physical objects or environment, but also the simple and static models or instantiation such as geometric models from CAX $\underline{x}$ software. All the needed information can be collected and transferred to virtual control layer by CPS.

\section{- Virtual Control Layer}

All decisions and control can be complete in this layer. The Control instructions are fed back to the physical layer through CPS.

Based on the multi-dimensional or multi-granularity heterogeneous static model and real-time data collected by the physical object layer, the physical execution units of each production process of the physical layer are mapped to the virtual layer by the twin modeling technology to reflect its real-time operation. According to the operational relationship corresponding to the physical layer, a virtual operation system is formed in the virtual layer, and the operation process of the physical layer is systematically and accurately mapped, at the same time, the OsC mechanism and synchronization mechanism should be imported to this layer, which makes it possible to control the production execution dynamically. 
The Virtual Control Layer contains three sub-levels: the twin model layer, the twin mapping layer, and the twin state layer (as shown in the upper part of Fig. 2).

Twin model layer: The basic for OsC is modeling the physical objects, which is the fundamental function of this layer. Various static models are formed at this level based on the raw data from the physical object layer. It contains a static model library of heterogeneous constituent units used to construct a general SPOS multi-scale operation mode. It not only includes the production resource model, but also includes the supply model, the relational model, the control model and the jamming model to support the linkage production operation control process of other models.

Twin image layer: Twin image layer instantiates the twin model layer based on the static data, and then combines the operational relationship and full element information to reflect the operational status of the physical layer in real time and provides decision information for the upper twin state layer. The basic image structure is instantiated by the corresponding twin models. Once the physical word in the execution process, this layer acquires full-feature real-time information from the physical layer through CPS and input the basic image structure to form a runtime SPOS twin image.

Twin state layer: The twin state layer introduces decision-making mechanisms such as OsC to make decisions and feed the results back to the physical layer In order to effectively judge the status of SPOS and control it, three basic state models that can satisfy the OsC mechanism are mapped in this layer, which are: (1) Multiscale twins is mapped to "Theoretical Optimal State" under the synchronized optimization of the theoretical model and the initial parameters. In this case, it can reflect the theoretical SPOS execution state and guide the production operation process as an initial plan, which is like the traditional production plan. (2) When SPOS is running, the "Actual State" can be formed by SPOS execution mapping to reflect the real-time operation state of SPOS. (3) When the dynamic interference from different factors occurs in the production operation process, the " Adaptive Optimal State " can be obtained based on the collaboration of SPOS total factor twin data and the multi-scale real-time twin mapping, which is used to guide the execution state of the next step of the SPOS.

To be effective, the digital twin control framework is not only required to digitize the physical world through CPS, but also a proper decision and control method. In this work, the OsC is used in virtual layer to make optimal decisions.

\subsection{The opti-state concept}

This work refers to the Opti-state concept proposed by our research team [48,49], and give a brief introduction on opti-state concept first.

\subsubsection{The definition of opti-state}

Key Performance Indicator, KPI is a quantitative management indicator of production system measurement process performance, such as production efficiency, production cost, customer satisfaction and so on. The KPI consists of various parameters, variables and constants that the system operates, such as machine speed, fuel and power costs. The state of measuring the operation of a production system can be derived from a 
combination of multiple KPIs, and the weight values between the KPIs are also different. The relationship between the state optimization of the production system and the KPI is that only the KPIs that are concerned can be classified as optimal targets of the optimization model, and some KPIs become the constraints of the model.

The "state" of the production system is a set of KPI values composed of system parameters, variables and these parameter variables at a certain moment. From the perspective of optimization, the state is a model set in which the system changes in time dimension. As the production system operates, the state of the system is constantly changing, including parameters, variables, KPI characterization values, and models.

The $\mathrm{OsC}$ is an online, active, timely and synchronization systematic control method that acts on the overall structure and synchronization operating parameters of the system. It can be used to make adaptive decisions to adjust the system to reach the current optimum state after the SPOS was affected by dynamics. Refer to the previous work in our research team, the opti-state of the production system can be explained from three states:

(1) Theoretical Opti-State: It based on the optimum structure and process before the production process. (2) Adaptive Opti-State: It is a state that the actual operating state can constantly approaching to after adaptive control and correction is made according to the random dynamics captured and the current resource state. (3) Actual State, that is, the real-time operating state of the production system.

\subsubsection{The opti-state control method}

After the SPOS is disturbed by uncertainties, the system has three control methods. The schematic diagram of the three control methods is as follows:

- Free state control method (FsC)

When system is disturbed by uncertainties, due to the lack of real-time information to control or lack of a reasonable decision-making method, the execution layer operates in a free state. This control method may result in the system being difficult to accomplish the stated target because it was not corrected in time. (as part A of Fig. 3)

- Responsive control method (RC)

alt-text: Fig 3

Fig. 3 


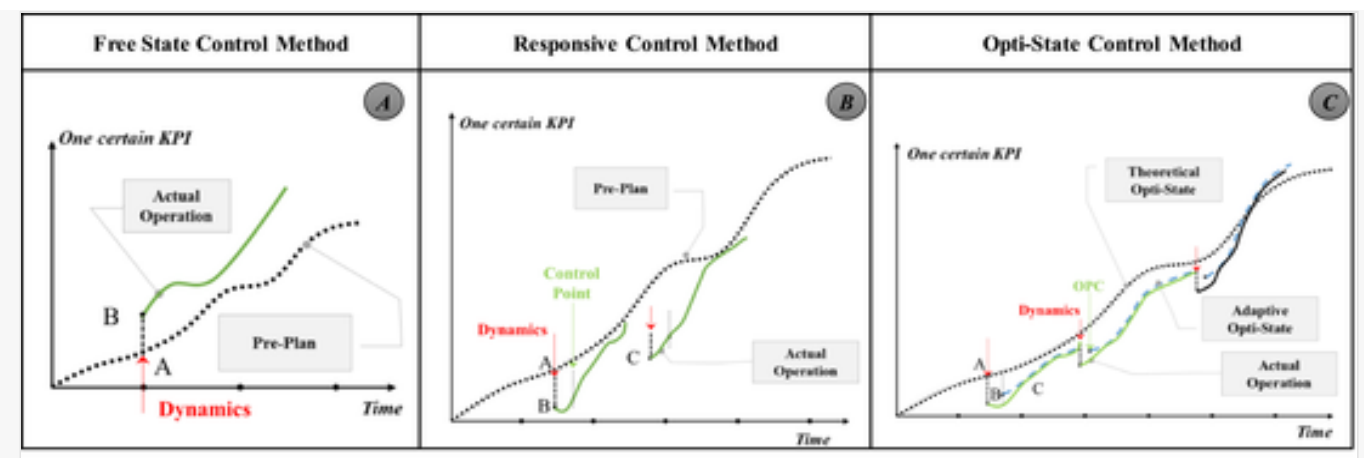

Three control methods.

When system is disturbed by element uncertainty factors, the system can adjust the structure or operating parameters to reduce the impact of interference by adopting a certain optimization method, such as synchronization optimization $[38,39]$ and resilient control, so as to achieve the original operational target. (as part B of Fig. 3)

- Opti-state control method (OsC)

When system is disturbed by target uncertainty factor, the $\mathrm{OsC}$ method can help decision-making layer recalculate an optimal target for the system and make an implementation plan under the new target for the executive layer. (as part $\mathrm{C}$ of Fig. 3)

The details about the logic of opti-state control is discussed in the previous work of authors' research team. This work just gives a brief introduction.

In the normal operation of the production system, the vertical dimension state, such as equipment parameters, system configuration, production plan, etc., remains stable, and the horizontal dimension state, such as production plan, tends to be consistent. When the SPOS is disturbed by uncertainties, a rapid and accurate decision can be made based on the real-time data and state from the production environment by decisionmaking layer, which is set an adaptive optimal state (as shown in the dotted line in Ppart C of Fig. 4). The system then guides the execution layer to continue operating according to the adaptive optimal state (point $\mathrm{C}$ ). The process reciprocates as the production runs.

alt-text: Fig 4

\section{Fig. 4}




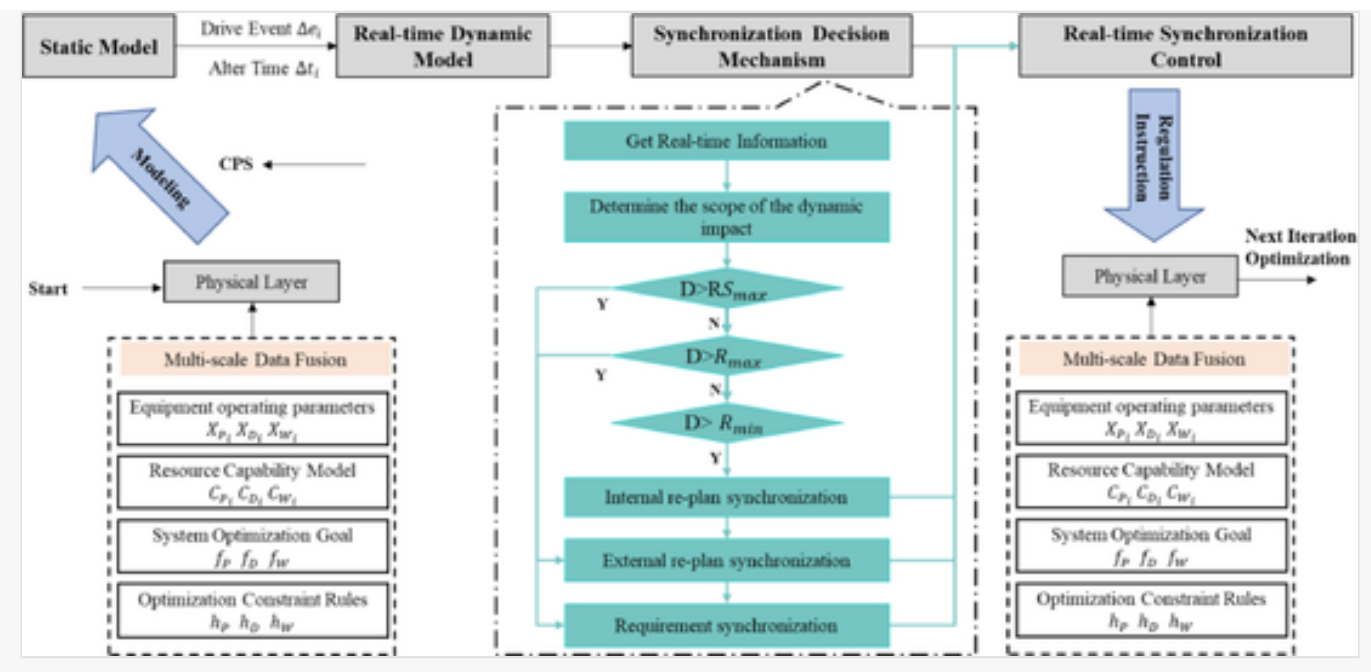

DT-based Mechanism process of synchronization control.

\subsection{Digital twin-based synchronization control mechanism: a qualitative method}

This section proposes the goal-preserving optimal state decision-making mechanism under the digital twin control system from the qualitative point of view, and introducing the synchronized decision-making mechanism proposed by my research team for the influence range of various dynamic factors [39].

Due to the application of digital twin-based control framework, the decision-making layer can obtain the information of the execution layer from coarse-grained static information to the multi-scale dynamic information. The decision-making layer is based on multi-scale dynamic information and it can achieve lean management under a system-level synchronization decision mechanism. Therefore, digital twin-based synchronization control mechanism promotes the decision-making method of complex large-scale system from "coarse-grained static local decision-making" to "fine dynamic synchronized decision-making", improving the precision of collaborative operation, reducing the synergy difference caused by dynamics and the coordinated operation cost, and ultimately achieving the optimal state operation of the system execution life cycle. The synchronized control decision mechanism is shown in Fig. 4:

Step 1. Based on the IoT perception technology and CPS, real-time sensing, acquisition and fusion of the shop to execute multi-scale and total factor information such as on-site hardware resources and software resources. Next, build a static model of the synchronized unit. At the same time, the quantitative multi-unit coordinated decision-making mechanism under the system optimal goal guidance and optimal rules constraints through the target cascade, parameter coupling, constraint association, inter-linkage parameter collaborative optimal decision to develop the initial operational plan;

Step 2. In the production execution phase, based on the basic static model formed by the initial plans, the virtual model layer driven by the driving event or transition time performs a complete mirror mapping of the real-time operation of the SOPS and constructs a real-time dynamic virtual model. At the same time, based on real-time production data, historical production data and other plant twin data, the SPOS execution process is monitored, simulated, evaluated or predicted. By comparing the actual operation results with the real-time system optimization state under the influence of uncertain factors, the multi-unit real-time synchronized 
decision mechanism is triggered by judging the influence range of the system based on the expected execution deviation value (the logic chart in the middle of Fig. 4):

(a) Decomposition of multi-dimensional optimal states to determine the dynamic impact on those optimal targets;

(b) Get real-time information on the execution layer;

(c) Determine the scope of the dynamic impact and the scope of coping ability of every unit, If $\mathrm{R}_{\min }<\mathrm{D}<\mathrm{R}_{\max }$, use internal re-plan synchronization;

(d) If $\mathrm{D}>\mathrm{R}_{\max }$, use external re-plan synchronization;

(e) If $\mathrm{D}>\mathrm{RS}_{\max }$, use requirement synchronization;

Step 3. In the real-time coordinated control phase: the decision result for the pre-order step is released to the production site. By introducing a superior state control mechanism, the physical layer resource configuration structure, equipment operating parameters, and shop floor planning are controlled in real time by means of control instructions to realize real-time online optimization of SPOS.

\subsection{ATC-based synchronization method: a quantitative method}

The multidisciplinary optimization method (MDO) is one kind of optimization method that using distributed computer network technology to integrate knowledge of multi disciplines, integrating the models and analysis tools of multi disciplines according to the idea of design-oriented in the whole design process of complex systems [50]. Through effective design and optimization of strategy organization and management design process, the overall optimal solution of the system can be obtained by making full use of the coordination effect produced by the interaction of various disciplines. As a new multidisciplinary optimization method, the target cascading analysis (ATC) method is used to solve the problem of distributed and decomposable into hierarchical architecture. The SPOS proposed in this paper is a typical distributed hierarchical decision structure. Fig. 5 shows a Ftypical synchronized decision model for ATC hierarchical production system.

\section{alt-text: Fig 5}

\section{Fig. 5}
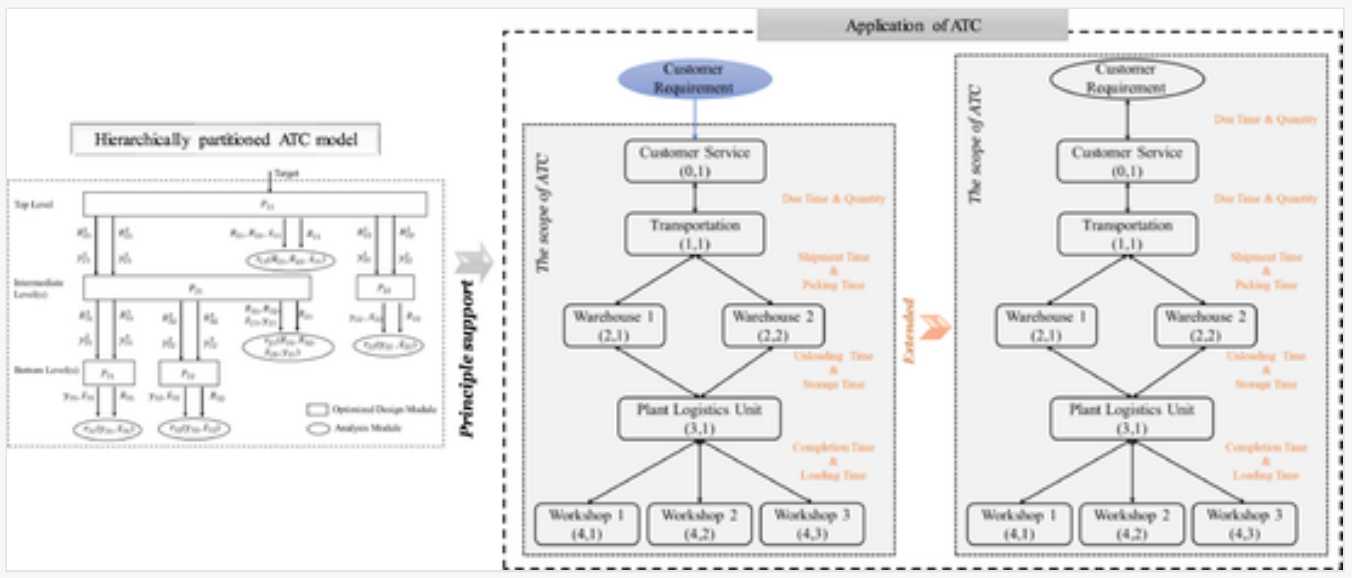
The ATC translating the overall design targets of a system to the specifications of the constituent elements that are decomposed to the multilevel hierarchy: each subsystem is associated with its parent system, which affect the synchronous execution of multi-workshop production plan and the synchronous completion of multibranch plant production tasks in the actual production process, thus affecting the dynamic real-time acquisition of complete order delivery, and ultimately achieving the optimization of the whole system through the dynamic coordination with the parent system. In the ATC-based hierarchical synchronized control model, two important modules are formed, which are optimized design module and analysis module [51]. The optimized design module is responsible for the optimization of the sub-goals, while the analysis module is used to calculate the subsystem response.

In the synchronized process of the hierarchical production system, the ATC synchronized coordination process is divided into the following steps:

Step 1: Establish a correct and hierarchical ATC target association model for SPOS in static situations;

Step 2: Indicate the key connections between the levels of the static system, including the response and the connection variables. The response is the shared amount between the parent subsystem elements. The connection variable is the shared amount of the subsystem elements. The consistency of the connection quantity is determined by its parent element. To coordinate;

Step 3: Establish a local objective function model for each layer of the system;

Step 4: According to the dynamics generated during the execution of the production system, the whole synchronized process is triggered. As the synchronized process moves forward to solve the dynamic synchronized goals of each layer system, the execution in the dynamic environment of the production system is promoted.

For the OsC method, in order to meet the optimal operating state of the SPOS, there may be cases where the system actively refuses to meet the requirement of customers. Therefore, this work incorporates customer requirements into the optimization model to calculate the optimal requirement that a system can meet. After that, the system performs ATC optimization according to the new system target and obtains quantitative control results. The detailed steps are similar to traditional ATC. The schematic diagram is shown in the right part of Fig. 5.

\section{Case Study}

This case study is conducted in a leading paint manufacturer in China. An information system for the production-logistics stage of this enterprise based on the $\mathrm{OsC}$ method proposed in this study has been implemented. This case illustrates the application model and actual effect of the OsC and synchronization control mechanism.

\subsection{Background and the workflow of case company}


As the top enterprise in the domestic industry, the market share of case company is always in the forefront. The company has a typical process, leading production capacity and over 10,000 kinds of products. Paint products are highly customized, different customers have different requirements for color, particle size and gloss of the same product. One customer order contains multiple product types; thus, it makes the enterprise need more than one workshop to complete cooperatively the production of an order. The operation process of the enterprise meets to the typical SPOS process (as shown in the Fig. 1), and it has four production workshops (each workshop contains a fixed capacity offline point), a transport fleet and two finished product warehouses, and it also using rent resources or outsourcing service.

This case study focuses on the main stage of this company which is production, transportation and warehousing. The purpose is to illustrate the advantages of $\mathrm{OsC}$ method proposed in this work by comparing the effect of the system dealing which the dynamic with $\mathrm{OsC}$ method, the responsive control method and free state control method when a new custom order is inserted.

The production mode of the company is intermittent production, which combines the characteristics of discrete and process production. Therefore, its characteristics are as follows: (1) The design and use of equipment are flexible, and it can produce different products; (2) Adopt batch production mode, and the single production capacity of the production line is limited by the volume of the equipment.; (3) Process parameters vary with product types; 4) Due to some process involve some chemical reactions, the production time is uncertain.

Most types of production are using make-to-order production method and one order can be split into multiple sub-orders and produced separately by each workshop. Table 2 is part of customer orders of the company in one day. Each customer order contains four kinds of products. After receiving customer orders, the enterprise assigns production tasks to corresponding workshops according to types of products (Table 3 shows the concurrent machine information for each production process in each workshop). The products are temporarily stored finished product buffer after completion. Forklift trucks pick up goods from buffers according to product completion time and transport the finished product to the corresponding warehouse according to the attributes of the goods. The mode of forklift trucks transportation is Milk-Run. Finished product warehouse allocation the location according to the arrival time of forklift truck, and kitting delivery according to the shipment date (Table 4 shows the aisle information). The 3PL company transports the goods. The main cost of each stage is shown in Table 5.

alt-text: Table 2

Table 2

The presentation of Tables and the formatting of text in the online proof do not match the final output, though the data is the same. To preview the actual presentation, view the Proof.

Customer order information. 


\begin{tabular}{|l|l|l|l|l|l|l|}
\hline 1 & 6 & 6 & 7 & 6 & 25 & $10: 30$ \\
\hline 2 & 8 & 10 & 9 & 8 & 35 & $11: 30$ \\
\hline 3 & 10 & 8 & 10 & 8 & 36 & $12: 00$ \\
\hline 4 & 10 & 12 & 16 & 12 & 50 & $14: 00$ \\
\hline 5 & 18 & 18 & 14 & 14 & 64 & $15: 00$ \\
\hline 6 & 15 & 12 & 15 & 15 & 57 & $17: 00$ \\
\hline 7 & 20 & 18 & 18 & 15 & 71 & $17: 00$ \\
\hline 8 & 18 & 15 & 20 & 18 & 71 & $18: 00$ \\
\hline 9 & 15 & 20 & 18 & 20 & 73 & $18: 00$ \\
\hline 10 & 18 & 16 & 17 & 18 & 69 & 13 \\
\hline
\end{tabular}

alt-text: Table 3

Table 3

(i) The presentation of Tables and the formatting of text in the online proof do not match the final output, though the data is the same. To preview the actual presentation, view the Proof.

Parallel machine information for workshop processes.

\begin{tabular}{|c|c|c|c|c|c|c|}
\hline \multirow{2}{*}{$\begin{array}{l}\text { Working } \\
\text { Procedure }\end{array}$} & \multirow{2}{*}{$\begin{array}{l}\text { Procedure } \\
\text { name }\end{array}$} & \multirow{2}{*}{$\begin{array}{l}\text { Parallel machine } \\
\text { Number }\end{array}$} & \multicolumn{4}{|c|}{ Unit processing time (hour) } \\
\hline & & & 1\# workshop & 2\# workshop & $\begin{array}{l}3 \# \\
\text { workshop }\end{array}$ & $\begin{array}{l}4 \# \\
\text { workshop }\end{array}$ \\
\hline 1 & Disperse & $1 / 2 / 3$ & $0.8 / 0.8 / 0.5$ & $0.7 / 0.8 / 06$ & $0.7 / 0.5 / 0.7$ & $0.6 / 0.6 / 0.5$ \\
\hline 2 & Grind & $4 / 5 / 6 / 7$ & $0.8 / 0.8 / 1.2 / 0.8$ & $1 / 0.8 / 1.2 / 0.8$ & $1 / 0.8 / 0.8 / 1.2$ & $0.8 / 1 / 0.8 / 1.2$ \\
\hline 3 & Toning & $8 / 9 / 10 / 11$ & $1.2 / 0.8 / 1.6 / 0.8$ & $0.8 / 0.8 / 1.2 / 1.4$ & $0.8 / 1 / 0.8 / 1$ & $1 / 1.2 / 0.8 / 1.2$ \\
\hline 4 & filter & $12 / 13$ & $0.4 / 0.8$ & $0.4 / 0.8$ & $0.4 / 0.4$ & $0.4 / 0.8$ \\
\hline 5 & Filling & $14 / 15$ & $0.4 / 0.4$ & $0.5 / 0.5$ & $0.4 / 0.6$ & $0.5 / 0.4$ \\
\hline
\end{tabular}

alt-text: Table 4

Table 4

(i) The presentation of Tables and the formatting of text in the online proof do not match the final output, though the data is the same. To preview the actual presentation, view the Proof. 
SHIP aisle information.

\begin{tabular}{|l|l|l|}
\hline Warehouse Number & Aisle Name & Aisle Capacity (tray) \\
\hline $1 \#$ & A1-A20 & 20 \\
\hline $2 \#$ & B1-B10 & 30 \\
\hline
\end{tabular}

alt-text: Table 5

Table 5

(i) The presentation of Tables and the formatting of text in the online proof do not match the final output, though the data is the same. To preview the actual presentation, view the Proof.

Production logistics cost information.

\begin{tabular}{|l|l|}
\hline Cost Categories & Cost (Yuan) \\
\hline Fixed cost of production & 2000 \\
\hline Unit hour processing cost & 400 \\
\hline Cost of storage warehouse & 30 \\
\hline Cost of offline point cache & 50 \\
\hline Penalty cost for delay of order & 50 \\
\hline Warehouse location rental cost & 3000 \\
\hline Working cost & 60 \\
\hline Equipment consumption cost & 30 \\
\hline Other cost & 10000 \\
\hline
\end{tabular}

Table 6.

alt-text: Table 6

Table 6

(i) The presentation of Tables and the formatting of text in the online proof do not match the final output, though the data is the same. To preview the actual presentation, view the Proof.

Assumption list. 


\begin{tabular}{|c|c|c|c|}
\hline Number & Problem assumption & Number & Problem assumption \\
\hline 1 & $\begin{array}{l}\text { A customer order contains a variety of products, different } \\
\text { products produced in different workshops }\end{array}$ & 2 & $\begin{array}{l}\text { Each workshop produces only one } \\
\text { product. }\end{array}$ \\
\hline 3 & One category product in one order is a production batch & 4 & $\begin{array}{l}\text { Each order batch can only produce by } \\
\text { one machine at same time }\end{array}$ \\
\hline 5 & $\begin{array}{l}\text { Each order has multiple production processes, and all orders } \\
\text { have the same process }\end{array}$ & 6 & $\begin{array}{l}\text { Only one order is processed by one } \\
\text { machine at the same time }\end{array}$ \\
\hline 7 & $\begin{array}{l}\text { There are several parallel machines in each production } \\
\text { process. }\end{array}$ & 8 & $\begin{array}{l}\text { Processing time includes setting time } \\
\text { and switching time }\end{array}$ \\
\hline 9 & $\begin{array}{l}\text { The processing time of different machines in each process is } \\
\text { different }\end{array}$ & 10 & $\begin{array}{l}\text { No interruption is allowed after an order } \\
\text { begins to produce }\end{array}$ \\
\hline 11 & $\begin{array}{l}\text { After the order is completed, it stored at the offline point } \\
\text { waiting for transportation to warehouse }\end{array}$ & 14 & $\begin{array}{l}\text { Only one forklift truck is arranged for } \\
\text { each milk-run to perform pick-up tasks }\end{array}$ \\
\hline 15 & $\begin{array}{l}\text { Each forklift truck carries out pick-up tasks according to the } \\
\text { set route to all production workshop off-line points. }\end{array}$ & 20 & $\begin{array}{l}\text { All products from the same customer } \\
\text { order cannot be shipped until they are } \\
\text { stored. }\end{array}$ \\
\hline 18 & $\begin{array}{l}\text { No more than two orders are stored in one aisle at the same } \\
\text { time }\end{array}$ & 19 & $\begin{array}{l}\text { we do not consider there is no } \\
\text { availability goods routes for orders } \\
\text { temporarily }\end{array}$ \\
\hline
\end{tabular}

\subsection{Problem assumption}

In order to make the problem generality, the assumptions are as follows:

\subsection{Mathematical models}

Through the analysis of SPOS operation, the ideal operation state is the synchronization operation among the independent parts. The specific performance is the consistency of the completion time of customers order, loading time and shipment time. The evaluating indicator on benefit is maximizing resource utilization rate and minimize cost to meet customer needs.

Therefore, in the design of each layer of ATC model, the system target is to minimize the total cost. Mathematical models are built with the order completion time, loading time and shipment time as well as the cumulative total cost of every layer as the coupling variables. The specific parameter symbols are shown in Table 7. 
The presentation of Tables and the formatting of text in the online proof do not match the final output, though the data is the same. To preview the actual presentation, view the Proof.

parametric symbol information table.

\begin{tabular}{|c|c|c|c|}
\hline Symbol & Describe & Symbol & Describe \\
\hline$H$ & Number of production workshops & $h$ & Workshop number, $h=1,2, \ldots, H$ \\
\hline$n$ & Quantity of orders to be processed & $i$ & $\begin{array}{l}\text { Order number } i=1,2, \ldots, n+\alpha T \mathrm{i}=1,2, \\
\ldots, \mathrm{n}+\alpha \mathrm{T}\end{array}$ \\
\hline$Q_{i}$ & Total quantity of order $i$ & $Q_{i}^{h}$ & The quantity of order $i$ in workshop $h$ \\
\hline$J$ & Total quantity of order processes & $j$ & $\begin{array}{l}\text { Procedure number of orders, } j=1,2, \ldots, J \\
\mathrm{~m}=1,2,3, \ldots, \mathrm{J}\end{array}$ \\
\hline$M_{j}$ & Quantity of parallel machines of processes $j$ & $k_{j}$ & $\begin{array}{l}\text { Machine number of procedures } j \\
k_{j}=1,2, \ldots, M_{j}\end{array}$ \\
\hline$D_{i}$ & Delivery time of order $i$ & $S_{i, j}$ & Procedure $j$ of order $i$ \\
\hline$h_{t, v}$ & Forklift truck $v$ carries out the pick-up task at time $t$ & $v$ & Forklift truck number, $v=1,2, \ldots, N_{v}$ \\
\hline$L_{v}$ & Rated capacity of vehicle $v$ & $T_{\max }$ & The latest work time everyday \\
\hline$N_{v}$ & Total quantity of forklift truck & $g_{i, t}^{h}$ & $\begin{array}{l}\text { Order } i \text { of workshop } h \text { is to be warehoused } \\
\text { at time } t\end{array}$ \\
\hline$N_{w a r}$ & Total quantity of aisle in & $l$ & Aisle number, $l=1,2, \ldots N_{w a r}$ \\
\hline$V_{l}$ & The capacity of aisle $l$ & $C_{p r o}^{h}$ & Production cost in unit time of workshop $h$ \\
\hline$C_{\text {tar }}$ & Unit time delay penalty cost & $C_{w a r}$ & Warehouse storage cost in per unit time \\
\hline$C_{b u f}^{h}$ & Unit time offline point caching cost of workshop $h$ & $C_{v}$ & Fixed distribution cost of forklift truck $v$ \\
\hline$C_{\text {war }}^{f i x}$ & Fixed cost for per warehouse & $C_{p}^{f i x}$ & Fixed cost of workshop production \\
\hline$T_{i, j, k_{j}}^{h}$ & $\begin{array}{l}\text { Unit process time of procedure } j \text { in workshop h on } \\
\text { machine } k_{j}\end{array}$ & $t_{o, i}^{h}$ & Offline time of order $i$ in workshop $h$ \\
\hline$t_{s_{i, j}}^{h}$ & Process $j$ start time of order $i$ in workshop $h$ & $t_{e_{i, j}}^{h}$ & $\begin{array}{l}\text { Processing end time of procedure } j \text { of order } i \\
\text { in workshop } h\end{array}$ \\
\hline$t_{\mathrm{tr}, \mathrm{i}}^{h}$ & Transportation time of order $i$ in workshop $h$ & $t_{\text {out }, i}^{h}$ & $\begin{array}{l}\text { The time of order } i \text { discharged from } \\
\text { workshop } h\end{array}$ \\
\hline$t_{S}$ & Service time of forklift truck & $t_{\mathrm{in}, \mathrm{i}}^{h}$ & The time of order $i$ stored from workshop $h$ \\
\hline$x_{i, j, k_{j}}$ & $\begin{array}{l}\text { Production stage decision variables, process } j \text { of order } i \text { is } \\
\text { processed by machine } k_{j}\end{array}$ & $T_{d e l_{i}}$ & Delayed delivery time of order $i$ \\
\hline out $_{t, i}$ & Warehousing stage decision variables, order $i$ is & $y_{i, p}$ & Production stage decision variables, order $i$ \\
\hline
\end{tabular}




\begin{tabular}{|c|c|c|c|}
\hline$l$ & discharged from aisle $l$ at time $t$ & & arranged process on location $\mathrm{p}$ \\
\hline$p_{t, i, l}$ & $\begin{array}{l}\text { When put } t, i, l=1 \text {, Represents the number of pallets } \\
\text { placed in aisle } l \text { for order } i \text { at time } t\end{array}$ & $p u t_{t, i, l}^{h}$ & $\begin{array}{l}\text { Warehousing stage decision variables, order } i \\
\text { is stored on aisle } l \text { at time } t\end{array}$ \\
\hline$D T$ & The moments when customer demand dynamics occur & $u_{l, t}$ & $\begin{array}{l}\text { The statement of aisle } l \text { at time } t, l \text { is used, } 0 \\
\text { is not used }\end{array}$ \\
\hline$T$ & Target cost of system level & $T C$ & Total cost of production-logistics system \\
\hline$\alpha_{T}$ & New orders dynamically added at time $t$ & $T_{\text {buf }}^{h}{ }_{i}^{h}$ & $\begin{array}{l}\text { The storage time of order } i \text { at the offline } \\
\text { point } h\end{array}$ \\
\hline$T_{\text {war } i}$ & The warehouse storage time of order $i$ & $P$ & Company total profit \\
\hline$R_{\text {total }}$ & Company total revenue & $C_{\text {total }}$ & Company total cost \\
\hline$p_{h}$ & Sales price of variety $h$ & & \\
\hline
\end{tabular}

\subsubsection{Warehouse scheduling model}

Warehouse unit arranges aisle for products. In order to keep the warehouse efficient, it must short the time of order in aisle as much as possible. At this model layer, turn the storage time to storage cost $T C_{\text {war }}$. As mentioned above, the difference of different products from same order extend the storage time. Thus, this model layer and transportation layer treat the loading time $t_{i n, i}^{h}$ as coupling variable. Mathematic model is as follows:

$\min :\|T C-T\|+\varepsilon^{0}$

$$
\sum_{h=1}^{H} \sum_{i=1}^{n}\left(w_{f}^{11}\right)^{2} \times\left(t_{i n, i}^{h}-t_{i n, i}^{h 1}\right)+\left(w_{f}^{12}\right)^{2} \times\left(T C_{t r a n}-T C_{t r a n}^{1}\right) \leq \varepsilon^{0}
$$

$$
T C=T C_{\text {tran }}+T C_{\text {war }}+T C_{\text {tar }}
$$

$$
T C_{w a r}=\sum_{l=1}^{N_{\text {war }}} u_{l, t} \times C_{w a r}^{f i x}+\sum_{h=1}^{H} \sum_{i=1}^{n}\left(t_{\text {out }, i}-t_{i n, i}^{h}\right) \times Q_{i}^{h} \times C_{w a r}
$$

$$
T C_{t a r}=\sum_{i=1}^{n} T_{d e l_{i}} \times C_{t a r} \times Q_{i}
$$




$$
Q_{i}=\sum_{h=1}^{H} Q_{i}^{h}, i=1,2, \ldots, n+\alpha_{T}
$$

$$
t_{\text {out }, i}=D_{i}
$$

$$
\sum_{l=1}^{N_{\text {war }}} u_{l, t} \leq N_{\text {war }}
$$

$$
\sum_{l=1}^{N_{\text {war }}} p u t_{t, i, l}^{h} \times p_{t, i, l}^{h}=Q_{i}^{h}
$$

$$
\sum_{a, b=1}^{n} p u t_{t, a, l}^{h} \times p_{t, a, l}^{h}+p u t_{t, b, l}^{h} \times p_{t, b, l}^{h} \leq V_{l}, l=1,2, \ldots, N_{w a r}
$$

$$
\sum_{i=1}^{n} p u t_{t, i, l}^{h} \leq 2, l=1,2, \ldots, N_{\text {war }}
$$

Eqs. (1.1) and (1.2) is optimization target of whole system; TC is total cost of system; T is the target of system; $\varepsilon^{0}$ is permissible error range of response at system level. $t_{i n, i}^{h}$ and $T C_{\text {tran }}$ is system layer setting loading time of orders for transportation layer and system target of total cost of transportation layer; $t_{i n, i}^{h 1}$ and $T C_{t r a n}^{1}$ are system response variables of transportation scheduling layer uploaded to system; Eq. (1.3) means that the total cost of the system layer consists of the cumulative cost of the transport layer, the storage cost and the delay cost; Formula(1.4)-(1.5) is storage cost and delaying cost; Eq. (1.6) is quantity of orders produced by manufacturing enterprises; Eq. (1.7) means the shipment time of orders is the delivery date. Eq. (1.8) is quantity of being used aisle at any time; Eq. (1.9) means the number of orders in stock equals the total number of orders. Eq. (1.10) is capacity constraints of aisle; Eq. (1.11) means no more than two orders can be placed in a single aisle at any time.

\subsubsection{Transportation scheduling model}

Plant logistics unit arranges forklift to transport products from different workshops to warehouses. In order to reduce the overstocking from finished production buffers, all finished sub-orders should be transferred to the 
warehouses as soon as possible. So, loading time should be as consistent as possible with complete time. Therefore, coupling variables which are belong to this layer model and production unit is products complete time $t_{o, i}^{h}$. The backlog cost of WIP caused by delaying transportation is $C_{b u f}^{h}$; Mathematic model is as follows:

$$
\min :\left(w_{f}^{11}\right)^{2} \times\left(T C_{t r a n}-T C_{\text {tran }}^{0}\right)^{2}+\left(w_{f}^{12}\right)^{2} \times \sum_{h=1}^{H} \sum_{i=1}^{n}\left(t_{i n, i}^{h}-t_{i n, i}^{h 0}\right)^{2}+\varepsilon^{1}
$$

$$
\sum_{h=1}^{H}\left[\sum_{i=1}^{n}\left(w_{h}^{21}\right)^{2}\left(t_{o, i}^{h}-t_{o, i}^{h 2}\right)+\left(w_{h}^{22}\right)^{2}\left(T C_{p}^{h}-T C_{p}^{h 2}\right)\right] \leq \varepsilon^{1}
$$

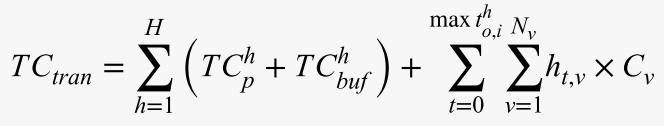

$T C_{b u f}^{h}=\sum_{i=1}^{n}\left(t_{i n, i}^{h}-t_{o, i}^{h}\right) \times C_{b u f}^{h}$

$$
Q_{b u f, t}^{h}=\sum_{0}^{t} \sum_{i=1}^{n} O_{t, i}^{h} \cdot Q_{i}^{h}-\sum_{0}^{t} \sum_{i=1}^{n} h_{t, i}^{h} \cdot Q_{i}^{h}
$$

$$
Q_{b u f, t}^{h} \leq Q_{b u f, m}^{h}
$$

$$
t_{t r, i}^{h}=g_{i, t}^{h} \cdot t
$$

$$
t_{i n, i}^{h}=t_{t r, i}^{h}+t_{s}
$$

$$
\sum_{t=0}^{\max t_{o, i}^{h}} g_{i, t}^{h}=1, i \in\left\{1,2, \ldots, n+\alpha_{T}\right\}, h \in\{1,2, \ldots, H\}
$$




$$
\sum_{v=1}^{N_{v}} h_{t, v} \leq 1
$$

$$
\sum_{h=1}^{H} \sum_{i=1}^{n}\left(g_{i, t}^{h} \times Q_{i}^{h}\right) \leq \sum_{v=1}^{N_{v}} h_{t, v} \times L_{v}, t \in t_{o, i}^{h}
$$

Eqs. (2.1) and (2.2) is a target function from transportation layer, $T C_{\text {tran }}$ and $t_{i n, i}^{h}$ is accumulated cost of transportation layer and loading time of every sub-orders, which are a target of the transportation layer settled from warehousing layer. $\varepsilon^{1}$ is error range. $t_{o, i}^{h}$ and $T C_{p}^{h}$ both are responses variables uploaded to this layer from the production layer. Eq. (2.3) is accumulated cost of transportation layer, which is consisted of finished production buffer $\operatorname{cost} C_{b u f}^{h}$, production $\operatorname{cost} C_{p r o}^{h}$ and assigning vehicle cost. Eq. (2.4) means the finished production buffer cost is proportional to the finished production buffer time. Eq. (2.5) means the order quantity in finished production buffer $h$ at time $t$ equals the difference between finished order number and inbound order number at time $t$. Eq. (2.6) means the number of products in finished production buffer cannot exceed the maximum capacity of the buffer. Eq. (2.7) means loading time of order i at workshop h; Eq. (2.8) means warehousing time i equals loading time plus vehicle servicing time; Eq. (2.9) means each order can only transporting by one vehicle; Eq. (2.10) means arrange at most one vehicle to transport at any minute; Eq. (2.11) means the quantity of orders of picking up by forklift truck cannot exceed the maximum capacity of vehicle.

\subsubsection{Production schedule model}

The production layer makes an executable schedule plan for every workshop, in addition, the schedule plan should minimize the compete time of sub-orders which belong to one custom order. Therefore, the complete time $t_{o, i}^{h}$ from every workshop pass to transportation layer as coupling variable. The target of the production layer is to produce all products in the shortest time. $x_{i, j, k_{j}}$ and $y_{i, q}$ both are decision variables for optimizing every sub-system of the production layer, and each of them can determine orders are processed at which machine in every workshop and sequence of processing order. The mathematic model is as follows:

$$
\min : \max C_{i, J}+\sum_{h=1}^{H}\left[\left(w_{h}^{21}\right)^{2} \times \sum_{i=1}^{n}\left(t_{o, i}^{h}-t_{o, i}^{h 1}\right)+\left(w_{h}^{22}\right)^{2} \times\left(T C_{p}^{h}-T C_{p}^{h 1}\right)\right]
$$

$$
T C_{p}^{h}=C_{p}^{f i x}+\sum_{i=1}^{n} \sum_{j=2}^{J}\left(t_{e_{i, j}}^{h}-t_{e_{i, j-1}}^{h}\right) \times C_{p r o}^{h}
$$




$$
t_{e_{i, j}}^{h}=t_{s_{i, j}}^{h}+\sum_{k_{j}}^{M_{j}} x_{i, j, k_{j}}^{h} \times T_{i, j, k_{j}}^{h} \times Q_{i}^{h}
$$

$$
t_{e_{i, j-1}}^{h} \leq t_{s_{i, j}}^{h}
$$

$$
t_{o, i}^{h}=t_{e_{i, J}}^{h}
$$

$$
\sum_{k_{j}=1}^{M_{j}} x_{i, j, k_{j}}^{h}=1, i \in\left\{1,2, \ldots, n+\alpha_{T}\right\}, j \in\{1,2, \ldots, J\}
$$

$$
\sum_{i=1}^{n} y_{i, q}^{h}=1
$$

Eq. (3.1) is the scheduling objective of the production layer, which is to minimize the maximum completion time and deviation from the upper layer. $C_{i, J}$ is the complete time of the last step of order $i . t_{o, i}^{h 1}$ and $T C_{p}^{h 1}$ are the scheduling targets set by the transport layer for the production layer. Eq. (3.2) is the sum of production cost, fixed production cost and processing cost. Eq. (3.3) is the completion time of the $j$ process of order $i$; Eq. (3.4) is the process order constraint, which means that the process must be completed after the previous process is completed; Eq. (3.5) is the order completion time is the completion time of the last process; Eq. (3.6) is a process that can only be processed on one machine at the same time; Eq. (3.7) is that each priority position in the order processing order can only correspond to one order.

\subsubsection{Total cost and revenue}

To simplify the problem, this case uses a simple and versatile total profit formula and selects the cost and revenue variables that are most relevant to this case.

$$
\mathrm{P}=R_{\text {total }}-C_{\text {total }}
$$

$$
R_{\text {total }}=\sum_{h=1}^{H} \sum_{i=1}^{n} Q_{i} \times p_{h}
$$




$$
C_{\text {total }}=\sum_{h=1}^{H} C_{p r o}^{h}+\sum_{h=1}^{H} \sum_{i=1}^{n} C_{b u f}^{h} \times T_{b u f_{i}^{h}}^{h}+\sum_{h=1}^{H} \sum_{i=1}^{n} C_{\text {war }} \times T_{\text {wari }}+C_{\text {ret }}+\omega \times R_{\text {total }}+C_{\text {oth }}
$$

Eq. (4.1) is the total profit of the company, Eq. (4.3) is the total produce revenue from all orders. Eq. (4.3) is the total cost, $\sum_{h=1}^{H} C_{p r o}^{h}$ is the production cost of all orders, $\sum_{h=1}^{H} \sum_{i=1}^{h} C_{b u f}^{h} \times T_{b u f_{i}^{h}}^{h}$ is the storage cost at offline point of all orders, $\sum_{h=1}^{H} \sum_{i=1}^{n} C_{w a r} \times T_{\text {wari }}$ is the warehouse storage cost of all orders, $C_{r e t}$ is resource rental cost, and $\omega \times R_{\text {total }}$ is raw material cost.

The fundamental target of a manufacturing enterprise is to ensure profitability. We set this target as the optimal operating state of the system, that is, the target of the OsC method. After the production system is affected by dynamics, we use the following steps to complete the OsC decision-making method: (1) The system re-plan using the RC method to accept all the new demand from customers. (2) Set different degrees of customer demand, compare the profitability of the system under different degrees of customer demand. (3) Re-planning using the customer demand degree when the system is most profitable.

\subsection{Result analysis}

The above examples are conducted by MATLAB 2017a on a PC with Intel(R) Core (TM) i7-3630QM CPU and 8GB RAM. The analysis models of the production and transportation layer subsystems are solved by Genetic Algorithm. The parameters of the genetic algorithm are set as follows: population size 50, iteration number 100 times, cross probability $P_{c}=0.6$, mutation probability $P_{m}=0.06$. The storage layer is solved using a rule-based heuristic algorithm. At the same time, the ATC coordination optimization method is used to realize the system-level coordination of multi-unit decision results. The results of the ATC-based decision model are used as input to the total revenue and cost model, and the revenue and cost under different dynamic degrees are calculated. The results are shown in Figs. 6 and 7.

alt-text: Fig 6

\section{Fig. 6}



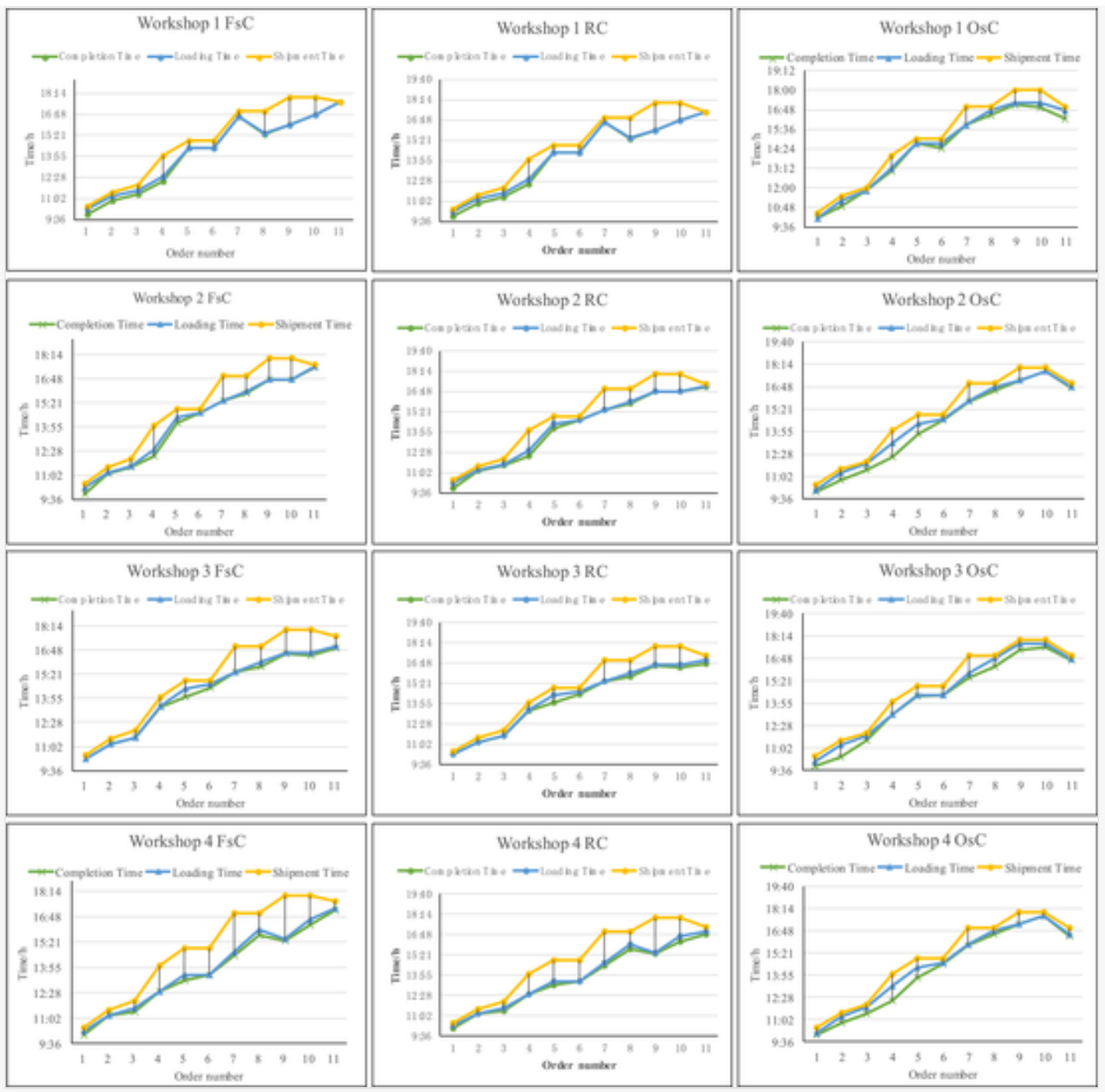

Time comparative results among $\mathrm{FsC}, \mathrm{RC}$ and $\mathrm{OsC}$.

\section{alt-text: Fig 7}

\section{Fig. 7}

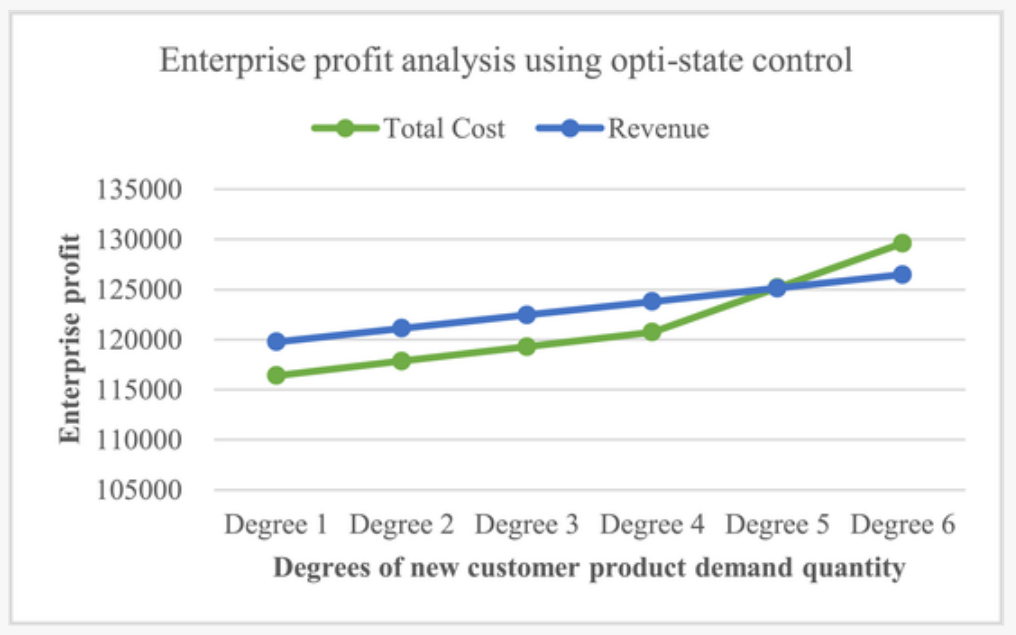

Enterprise profit analysis using OsC. 
The warehouse storage time and offline storage time can reflect the efficiency of the transport and storage unit. Fig. 6 shows that during the operation of the production system, a new customer order with a demand of 12, 6 , 6, and 12 for each workshop was inserted. In response to this dynamic, the system makes a new decision for each order of new finished product complete time $t_{o, i}^{h}$, transportation time $t_{\mathrm{tr}, \mathrm{i}}^{h}$ and shipment time $t_{\text {out }, i}^{h} \cdot t_{o, i}^{h}, t_{\mathrm{tr}, \mathrm{i}}^{h}$ and $t_{\mathrm{out}, i}^{h}$ are the calculation results of the ATC model. $T_{b u f}^{h}=t_{\mathrm{tr}, \mathrm{i}}^{h}-t_{o, i}^{h}$ and $T_{\text {wari }}=t_{\mathrm{out}, i}^{h}-t_{\mathrm{tr}, \mathrm{i}}^{h}$.

As discussed in Section 4.2.2, there are 3 ways to response to this uncertainty. Here we compare the effect of free state control method and responsive control method. It is clearly that the buffer waiting time and the instock time of most workshops are shorter after responsive control method is adopted, which proved that the using a synchronization control method is more effective.

The primary goal for an enterprise is ensure profitability, from the perspective of system revenue, OsC judges whether the stated decision target (to fully meet the requirement of new customer) is reasonable. This study analyzes the profit of enterprise, as shown in Fig. 7, the horizontal axis is different degrees of new customer requirement, the vertical axis is the profit. Table 8 shows different degrees of new customer requirement.

alt-text: Table 8

Table 8

(i) The presentation of Tables and the formatting of text in the online proof do not match the final output, though the data is the same. To preview the actual presentation, view the Proof.

New customer requirement degrees.

\begin{tabular}{|l|l|l|l|l|l|l|}
\hline & Degree 1 & Degree 2 & Degree 3 & Degree 4 & Degree 5 & Degree 6 \\
\hline Workshop 1 & 2 & 4 & 6 & 8 & 10 & 12 \\
\hline Workshop 2 & 1 & 2 & 3 & 4 & 5 & 6 \\
\hline Workshop 3 & 1 & 2 & 3 & 4 & 10 & 6 \\
\hline Workshop 4 & 2 & 4 & 6 & 8 & 12 \\
\hline
\end{tabular}

The Company invest different resource costs to meet different levels of customer demand. Degree 6 means that the company fully accepts the new order demand from the customer. In this case, an additional warehouse is introduced to store the products in accordance with the full acceptance of customer needs, thereby increasing the total cost. The same situation also occurs at degree 5. The extra cost even makes the total revenue of the company negative. Conversely, if the company take degree 4 as the new custom requirement the company can remain profitable. Then a new synchronization decision is made for the new customer demand with degree 4.

By comparing the $\mathrm{RC}$ and the $\mathrm{OsC}$ method, from a production perspective, using the $\mathrm{RC}$ method allows companies to meet customer needs at the lowest cost. Hence, from the perspective of enterprise-level 
profitability, the use of OsC method can help company make an optimal decision. Therefore, OsC method can keep the production system operating at an optimal state from a system level perspective.

\section{Conclusions}

This work proposes a digital twin-based opti-state control method for a synchronized production operation system. Aiming to keep the system operate in an optimal state after uncertainties interference, this method helps the decision-making layer of SPOS make an optimal decision online and autonomously based on the information acquired real-time, comprehensive and accurate under the framework of digital twin. Through the implementation of the case company, the effectiveness of $\mathrm{OsC}$ method is proved. This method provides a feasible implementation framework and means for SPOS online collaborative operation and autonomous decision control. The main contribution of this work is as below:

(1) By analyzing the characteristics of the general production system, the definition of SPOS is proposed and its characteristics are analyzed. Besides, uncertainties encountered by SPOS are also classified, then distinguishing the range of resilient control and opti-state control by the angle of uncertainty factors action point.

(2) By analyzing the link between OsC concept and digital twin, this study extends the basic concept of digital twin and proposed a digital twin-based opti-state control framework for SPOS.

(3) Based on the OsC concept put forward by the authors' research team, this work further elaborates the definition of "state" for a production system and enriches the definition of OsC from both qualitative and quantitative aspects: 1) From qualitative aspect, comparing another two different control methods of free state control and responsive control, and $\mathrm{OsC}$ and mechanisms are discussed in depth; 2) From quantitative aspect, incorporating customer needs into the ATC solution architecture, which give a new opti-state control idea.

Although this paper put forward the concept and control mechanism of opti-state, the mathematical modeling for opti-state need to be discussed in future work. Besides, the method of determining the target of OsC is also the focus of future research.

\section{Declaration of Competing Interest}

All authors have participated in (a) conception and design, or analysis and interpretation of the data; (b) drafting the article or revising it critically for important intellectual content; and (c) approval of the final version.

This manuscript has not been submitted to, nor is under review at, another journal or other publishing venue.

The authors have no affiliation with any organization with a direct or indirect financial interest in the subject matter discussed in the manuscript.

\section{Acknowledgment}


This work is supported by National Natural Science Foundation of China (51875251), 2018 Guangzhou Innovation Leading Talent Program, China (201909010006), Blue Fire Project (Huizhou) Industry-UniversityResearch Joint Innovation Fund of Ministry of Education, China (CXZJHZ201722), and the Fundamental Research Funds for the Central Universities, China (11618401).

\section{References}

The corrections made in this section will be reviewed and approved by journal production editor.

[1] P. Zawadzki, K. Żywicki, Smart product design and production control for effective mass customization in the Industry 4.0 concept, Manag. Prod. Eng. Rev. 7 (3) (2016) 105-112.

[2] J.-Q. Li, et al., Industrial internet: a survey on the enabling technologies, applications, and challenges, IEEE Commun. Surv. Tutor. 19 (3) (2017) 1504-1526.

[3] F. Tao, et al., Digital twin and its potential application exploration, Comput. Integr. Manuf. Syst 24 (1) (2018) 1-18.

[4] C.G. Rieger, D.I. Gertman, M.A. McQueen, Resilient control systems: next generation design research, Proceedings of the 2009 2nd Conference on Human System Interactions, IEEE, 2009.

[5] D. Bandyopadhyay, J. Sen, Internet of things: Applications and challenges in technology and standardization, Wirel. Pers. Commun. 58 (1) (2011) 49-69.

[6] T. Qu, et al., System dynamics analysis for an Internet-of-Things-enabled production logistics system, Int. J. Prod. Res. 55 (9) (2017) 2622-2649.

[7] S. Ren, et al., A comprehensive review of big data analytics throughout product lifecycle to support sustainable smart manufacturing: A framework, challenges and future research directions, J. Clean. Prod. 210 (2019) 1343-1365.

[8] K. Ding, P. Jiang, S. Su, RFID-enabled social manufacturing system for inter-enterprise monitoring and dispatching of integrated production and transportation tasks, Rob. Comput. Integr. Manuf. 49 (2018) 120-133.

[9] R.Y. Zhong, et al., RFID-enabled real-time manufacturing execution system for masscustomization production, Rob. Comput. Integr. Manuf. 29 (2) (2013) 283-292.

[10] P.J. Parikh, R.D. Meller, A travel-time model for a person-onboard order picking system, Eur. J. Oper. Res. 200 (2) (2010) 385-394.

[11] Y. Zhang, Z. Zhu, J. Lv, CPS-based smart control model for shopfloor material handling, IEEE Trans. Ind. Inf. 14 (4) (2017) 1764-1775. 
[12] K. Ding, et al., Hidden Markov model-based autonomous manufacturing task orchestration in smart shop floors, Rob. Comput. Integr. Manuf. 61 (2020) 101845.

[13] G.N. Schroeder, et al., Digital twin data modeling with automationml and a communication methodology for data exchange, IFAC-PapersOnLine 49 (30) (2016) 12-17.

[14] A. Moreno, et al., Virtualisation process of a sheet metal punching machine within the Industry 4.0 vision, Int. J. Interact. Des. Manuf. (IJIDeM) 11 (2) (2017) 365-373.

[15] T.H.-J. Uhlemann, et al., The digital twin: Demonstrating the potential of real time data acquisition in production systems, Procedia Manuf. 9 (2017) 113-120.

[16] X.V. Wang, et al., Human-robot collaborative assembly in cyber-physical production: Classification framework and implementation, CIRP Ann. 66 (1) (2017) 5-8.

[17] Y. Bazilevs, et al., Isogeometric fatigue damage prediction in large-scale composite structures driven by dynamic sensor data, J. Appl. Mech. 82 (9) (2015) 091008.

[18] Y. Cai, et al., Sensor data and information fusion to construct digital-twins virtual machine tools for cyber-physical manufacturing, Proc. Manuf. 10 (2017) 1031-1042.

[19] F. Tao, M. Zhang, Digital twin shop-floor: a new shop-floor paradigm towards smart manufacturing, IEEE Access 5 (2017) 20418-20427.

[20] Y. Zhang, et al., Agent and cyber-physical system based self-organizing and self-adaptive intelligent shopfloor, IEEE Trans. Ind. Inf. 13 (2) (2017) 737-747.

[21] K. Ding, et al., Defining a Digital Twin-based Cyber-Physical Production System for autonomous manufacturing in smart shop floors, Int. J. Prod. Res. (2019) 1-20.

[22] C. Zhuang, J. Liu, H. Xiong, Digital twin-based smart production management and control framework for the complex product assembly shop-floor, Int. J. Adv. Manuf. Technol. 96 (1-4) (2018) 1149-1163.

[23] R. Söderberg, et al., Toward a Digital Twin for real-time geometry assurance in individualized production, CIRP Ann. 66 (1) (2017) 137-140.

[24] P.D.U. Coronado, et al., Part data integration in the Shop Floor Digital Twin: Mobile and cloud technologies to enable a manufacturing execution system, J. Manuf. Syst. 48 (2018) 25-33.

[25] F. Tao, et al., Theories and technologies for cyber-physical fusion in digital twin shop-floor, Comput. Integr. Manuf. Syst. 23 (20) (2017) 1603-APA $\underline{1611 .}$

[26] F. Tao, et al., Digital twin workshop: a new paradigm for future workshop, Comput. Integr. Manuf. Syst. 23 (1) (2017) 1-9. 
M.A. Jing, Y.E. Yong, J.I.A. Qiusheng, Review of resilient control, Inf. Control 44 (1) (2015) $67-75$.

[28] K. Ji, D. Wei, Resilient control for wireless networked control systems, Int. J. Control Autom. Syst. 9 (2) (2011) 285-293.

[29] L. Cheng, et al., Integrated design of machine body and control algorithm for improving the robustness of a closed-chain five-bar machine, IEEE/ASME Trans. Mechatron. 17 (3) (2012) $587-591$.

[30] H.M. Soliman, M.H. Soliman, M.F. Hassan, "Resilient guaranteed cost control of a power system, J. Adv. Res. 5 (3) (2014) 377-385.

[31] X. Jin, A. Ray, R.M. Edwards, Integrated robust and resilient control of nuclear power plants for operational safety and high performance, IEEE Trans. Nucl. Sci. 57 (2) (2010) 807-817.

[32] W.-C. Lin, K.R.E. Villez, H.E. Garcia, Experimental validation of a resilient monitoring and control system, J. Process Control 24 (5) (2014) 621-639.

[33] J. Wang, et al., A novel resilient scheduling paradigm integrating operation and design for manufacturing systems with uncertainties, Enterp. Inf. Syst. 13 (4) (2018 $\underline{2019})+\underline{430}-18 \underline{447}$.

[34] D.D. Woods, Four concepts for resilience and the implications for the future of resilience engineering, Reliab. Eng. Syst. Saf. 141 (2015) 5-9.

[35] L. Monostori, et al., Cooperative control in production and logistics, Ann. Rev. Control 39 (2015) $12-29$.

[36] Y. Yang, J.F. Bard, Internal mail transport at processing \& distribution centers, IISE Trans. 49 (3) (2017) 285-303.

[37] G. Liotta, T. Kaihara, G. Stecca, Optimization and simulation of collaborative networks for sustainable production and transportation, IEEE Trans. Ind. Inf. 12 (1) (2016) 417-424.

[38] T. Qu, et al., IoT-based real-time production logistics synchronization system under smart cloud manufacturing, Int. J. Adv. Manuf. Technol. 84 (1-4) (2016) 147-164.

[39] T. Qu, et al., Internet of Things-based real-time production logistics synchronization mechanism and method toward customer order dynamics, Trans. Inst. Meas. Control 39 (4) (2017) 429-445.

[40] H. Luo, et al., Synchronized production and logistics via ubiquitous computing technology, Rob. Comput. Integr. Manuf. 45 (2017) 99-115.

[41] G. Zhang, et al., Extending augmented Lagrangian coordination for the optimal configuration of cloud-based smart manufacturing services with production capacity constraint, Rob. Comput. Integr. Manuf. 58 (2019) 21-32. 
[42] Y. Zhang, et al., A framework for smart production-logistics systems based on CPS and industrial IoT, IEEE Trans. Ind. Inf. 14 (9) (2018) 4019-4032.

[43] Balasubramanian, J. "Optimization models and algorithms for batch process scheduling under uncertainty", (2004): 1364-1364.

[44] H.-J. Zimmermann, An application-oriented view of modeling uncertainty, Eur. J. Oper. Res. 122 (2) (2000) 190-198.

[45] S. Subrahmanyam, J.F. Pekny, G.V. Reklaitis, Design of batch chemical plants under market uncertainty, Ind. Eng. Chem. Res. 33 (11) (1994) 2688-2701.

[46] E.N. Pistikopoulos, Uncertainty in process design and operations, Comput. Chem. Eng. 19 (1995) 553-563.

[47] J. Bräutigam, C. Esche, A. Mehler-Bicher, Uncertainty as a key value driver of real options, Proceedings of the 7th Annual Real Options Conference, 2003.

[48] T. Qu, K. Zhang, M. Yan, H. Guo, G.Q. Huang, C. Li, X. Li, Synchronized decision-making and control method for opti-state execution of dynamic production systems with internet of things, J. Mech. Eng. 54 (16) (2018) 24-33.

[49] K. Zhang, et al., IoT-enabled dynamic lean control mechanism for typical production systems, J. Amb. Intell. Human. Comput. 10 (3) (2019) 1009-1023.

[50] T. Qu, et al., Extending analytical target cascading for optimal supply chain network configuration of a product family, Int. J. Comput. Integr. Manuf. 22 (11) (2009) 1012-1023.

[51] H.M. Kim, Target Cascading in Optimal System Design, Diss. University of Michigan, 2001.

\section{Highlights}

- Consolidate the core requirements for intelligent manufacturing models

- The opti-state control (OsC) method helps keep the system adjust to the currently achievable optimal operating state when uncertainties affect the system

- Extend the basic framework of Digital Twin to the DT-based opti-state control framework 